\title{
Editorial
}

\section{Point-of-Care Ultrasound: The Future is Now}

Mark D Weber, ${ }^{1}{ }_{R N}, C R N P-A C, F C C M$, Sam Rosenblatt, ${ }^{1}{ }_{M D}, M S E d$, Thomas Conlon, ${ }^{1}{ }_{M D}$

Roughly 2 decades ago, the American College of Emergency Physicians published its initial guidelines for point-of-care ultrasound (POCUS). ${ }^{1}$ Since then, POCUS has expanded to the critical care setting and improved the quality and efficiency of care provided to our sickest patients. ${ }^{2}$ In this issue of the Annals of the Academy of Medicine, Singapore, Lau YH et al surveyed critical care physicians from 6 different teaching hospitals within Singapore, and inquired about their use and training in POCUS. ${ }^{2}$ The authors found that ultrasound was available (100\%), that diagnostic studies were being routinely performed (95.5\%), and that variations of training processes existed and were pursued by critical care providers. Translating training to effective care exposes institutions, providers and patients to risks. Respondents acknowledged that there was a lack of expert supervision and local resources, a lack of institutional and national accreditation processes, and an overall lack of guidance from the larger critical care POCUS community.

The lack of structured POCUS training and implementation is not a problem unique to Singapore. A survey of fellowship directors in the United States revealed that, while most institutions had an ultrasound machine, only $44 \%$ followed specific curricula to train their critical care fellows. Most of the programmes had limited number of faculty who were able to provide adequate mentoring for hands-on training in POCUS. ${ }^{3}$ In a survey of 114 intensive care units in Australia, Yang et al. identified key hurdles to the implementation of POCUS, including the lack of an organised training programme and a limited amount of time to apply towards education. ${ }^{4}$ Roughly $30-41 \%$ of intensivists who responded to the survey were untrained in the basic components of POCUS. Clearly, initiating and sustaining local POCUS training is an international challenge requiring attention.
To build these local training programmes, we must first agree on what constitutes "adequate training", given a historical lack of uniformity in this definition. ${ }^{5}$ Additionally, ill-defined competencies have led to challenges in creating a uniformly accepted educational platform. Thankfully, societies have begun to clearly delineate and define the competencies required for adequate POCUS training. ${ }^{1,6-8}$ These guidelines provided a scope of practice, identifying appropriate applications of ultrasound and their respective educational objectives. They also inform credentialing and integration of POCUS into professional practice, guiding centres in the creation and implementation of their individual POCUS programmes, including education on POCUS. ${ }^{9,10}$

As an example of how to utilise published guidelines, our own group modified POCUS core applications from the American College of Emergency Physicians guidelines to develop a 2-day local educational programme for paediatric critical care physicians. ${ }^{1,9}$ This training was delivered as a component of an institutional credentialing pathway, developed through multidisciplinary collaboration between critical care, cardiology and radiology specialists. Image archiving, documentation and quality assurance processes were developed. Programme and institutional leadership developed an annual maintenance of certification process resulting in $100 \%$ of paediatric critical care faculties credentialed in at least one core POCUS application (data not published). This approach of a didactic educational component combined with local structured programme build can help to define and achieve competence in critical care POCUS.

We should also ask the question of whether POCUS is an adjunct technology for a critical care physician or part of what it is to be a critical care physician. Current trainees in critical care disciplines frequently have exposure to POCUS within their earliest medical training.

\footnotetext{
${ }^{1}$ Department of Anesthesiology and Critical Care Medicine, Children's Hospital of Philadelphia, United States

Address for Correspondence: Mr Mark Weber, Children's Hospital of Philadelphia, Division of Anesthesiology and Critical Care Medicine, 3401 Civic Center Blvd. Philadelphia PA 19104, United States

Email: weberm@email.chop.edu
} 
Therefore, the need for formal external courses to teach basic POCUS will likely reduce over time. Ultrasound is becoming as ubiquitous as the stethoscope in many institutions and this may preclude the need for more formalised external agency certification processes. If POCUS is a component of what it is to be a critical care physician, national training platforms should formalise POCUS curriculum for trainee critical care providers. We need to acknowledge that not all (in fact, few) senior critical care clinicians will be expert educators and will require their own training processes. Thus, both external and internal training programmes will be required for the foreseeable future.

While this study had limitations, such as the low survey response rate $(41 \%)$ and participation bias, the authors did an excellent job reminding us that we cannot forget to support the ongoing implementation of new tools and technologies. It is not enough to simply invest in the technologic platform and tell clinicians to acquire images. Individual skill development requires standardised training as well as programmatic support through image storage and documentation solutions, quality assurance processes, and institutional credentialing to ensure that providers maintain competency in their clinical applications. Institutional investment in individual training and programmatic structuring will reap the benefits of improved clinical translation of skills, as well as measurable improvement in patient outcomes. Local, national and international POCUS communities need to work together to standardise basic critical care training platforms, both for emerging trainees as well as more senior faculties with less exposure to POCUS applications.

\section{REFERENCES}

1. American College of Emergency Physicians: Ultrasound Guidelines: Emergency, Point-of-care, and Clinical Ultrasound Guidelines in Medicine. Available from: https://www.acep.org/globalassets/new-pdfs/ policy-statements/ultrasound-guidelines---emergency-point-of-care-andclinical-ultrasound-guidelines-in-medicine.pdf.

2. Loh YH, Fong WK, Shahla Siddiqui, Tan CK, Jonathan JE Tan, Claudia JC Tien, KC See; Point-of-care ultrasound training among intensivists in Singapore: a multicentre survey. Annals, Academy of Medicine, Singapore. 2020. Ann Acad Med Singap. 2020;49:628-37.

3. Mosier JM, Malo J, Stolz LA, Bloom JW, Reyes NA, Snyder LS, et al. Critical care ultrasound training: a survey of US fellowship directors. J Crit Care. 2014 Aug;29(4):645-9.

4. Yang Y, Royse C, Royse A, Williams K, Canty D. Survey of the training and use of echocardiography and lung ultrasound in Australasian intensive care units. Crit Care. 2016 Oct 24;20(1):339.

5. Wong A, Galarza L, Duska F. Critical Care Ultrasound: A Systematic Review of International Training Competencies and Program. Crit Care Med. 2019 Mar;47(3):e256-e62.

6. Singh Y, Tissot C, Fraga MV, Yousef N, Cortes RG, Lopez J, et al. International evidence-based guidelines on Point of Care Ultrasound (POCUS) for critically ill neonates and children issued by the POCUS Working Group of the European Society of Paediatric and Neonatal Intensive Care (ESPNIC). Crit Care. 2020 Feb 24;24(1):65.

7. Expert Round Table on Ultrasound in ICU. International expert statement on training standards for critical care ultrasonography. Intensive Care Med. 2011 Jul;37(7):1077-83

8. Frankel HL, Kirkpatrick AW, Elbarbary M, Blaivas M, Desai H, Evans D, et al. Guidelines for the Appropriate Use of Bedside General and Cardiac Ultrasonography in the Evaluation of Critically Ill Patients-Part I: General Ultrasonography. Crit Care Med. 2015 Nov;43(11):2479-502.

9. Conlon TW, Himebauch AS, Fitzgerald JC, Chen AE, Dean AJ, Panebianco $\mathrm{N}$, et al. Implementation of a pediatric critical care $\mathrm{f}$ ocused bedside ultrasound training program in a large academic PICU. Pediatr Crit Care Med. 2015 Mar;16(3):219-26.

10. Arntfield RT. The utility of remote supervision with feedback as a method to deliver high-volume critical care ultrasound training. J Crit Care. 2015 Apr;30(2):441 e1-6. 